\title{
The occurrence of adverse drug reactions reported for attention deficit hyperactivity disorder (ADHD) medications in the pediatric population: a qualitative review of empirical studies
}

\author{
This article was published in the following Dove Press journal: \\ Neuropsychiatric Disease and Treatment \\ 15 December 2011 \\ Number of times this article has been viewed
}

\author{
Lise Aagaard ${ }^{1-3}$ \\ Ebba Holme Hansen ${ }^{1-3}$ \\ 'Department of Pharmacology and \\ Pharmacotherapy, Section for Social \\ Pharmacy, Faculty of Pharmaceutical \\ Sciences, University of Copenhagen, \\ Denmark; ${ }^{2}$ FKL-Research Centre for \\ Quality in Medicine Use, Copenhagen, \\ Denmark; ${ }^{3}$ Danish Pharmacovigilance \\ Research Project (DANPREP), \\ Copenhagen, Denmark
}

Background: To review empirical studies of adverse drug reactions (ADRs) reported to be associated with the use of medications generally licensed for treatment of attention deficit hyperactivity disorder (ADHD) symptoms in the pediatric population.

Methods: PubMed, Embase, and PsycINFO ${ }^{\circledR}$ databases were searched from origin until June 2011. Studies reporting ADRs from amphetamine derivates, atomoxetine, methylphenidate, and modafinil in children from birth to age 17 were included. Information about ADR reporting rates, age and gender of the child, type, and seriousness of ADRs, setting, study design, ADR assessors, authors, and funding sources were extracted.

Results: The review identified 43 studies reporting ADRs associated with medicines for treatment of ADHD in clinical studies covering approximately 7000 children, the majority of 6- to 12-year-old boys, and particularly in the United States of America (USA). The most frequently reported ADRs were decrease in appetite, gastrointestinal pain, and headache. There were wide variations in reported ADR occurrence between studies of similar design, setting, included population, and type of medication. Reported ADRs were primarily assessed by the children/their parents, and very few ADRs were rated as being serious. A large number of children dropped out of studies due to serious ADRs, and therefore, the actual number of serious ADRs from use of psychostimulants is probably higher. A large number of studies were conducted by the same groups of authors and sponsored by the pharmaceutical companies manufacturing the respective medications.

Conclusion: Reported ADRs from use of psychostimulants in children were found in clinical trials of short duration. Since ADHD medications are prescribed for long-term treatment, there is a need for long-term safety studies. The pharmaceutical companies should make all information about ADRs reported for these medications accessible to the public, and further studies are needed on the impact of the link between researchers and the manufacturers of the respective products. Keywords: adverse drug reactions, attention deficit hyperactivity disorder, children, pharmacovigilance

\section{Introduction}

Psychostimulants, such as amphetamine derivates, methylphenidate, and modafinil, as well as the nonstimulant medication atomoxetine, are considered first-line medication treatment of attention deficit hyperactivity disorder (ADHD) symptoms in the pediatric population. ${ }^{1}$ Case reports on serious cardiovascular adverse drug reactions (ADRs), sudden death, and psychiatric disorders led regulatory agencies to warn against the use 
of methylphenidate in the pediatric population in 2006 and $2007 .^{2,3}$ In 2006, warnings were also linked to atomoxetine use due to reports of hepatotoxicity and suicidal thoughts in children. ${ }^{4}$ Concern has been raised about ADRs from longterm treatment with ADHD medications, such as psychosis, sensitization, dependency, and withdrawal reactions. ${ }^{1}$ The issue of appropriate warnings about possible ADRs to the use of methylphenidate and other ADHD medications is ever more important as usage continues to increase rapidly in many countries: an increase in the number of treated patients has been observed, as well as an increase in the average dispensed daily dose of psychostimulants. ${ }^{5}$

The use of psychostimulants, particularly methylphenidate, to treat ADHD symptoms in children has increased rapidly since the 1990s. Studies have shown that the prevalence of psychostimulant use in children in the Netherlands increased eight times from 1996 to $2006,{ }^{6}$ and in Germany, prescription rates of methylphenidate increased by $96 \%$ from 2000 to 2007. ${ }^{7}$ From 1994 to 2004, the prevalence of psychostimulant use in Norwegian children increased five times, ${ }^{8}$ while the prevalence of stimulant medication increased ten times in American children from 1987 to $1996 .{ }^{9}$ Previous meta-analyses and reviews that evaluated the shortterm efficacy of psychostimulants on ADHD symptoms in children concluded that psychostimulants are more effective than placebo with respect to treating disturbed attention and impulsivity. ${ }^{1,10}$ Several articles have reported information about the safety of methylphenidate and other psychostimulants in clinical studies, ${ }^{11}$ but to the current reviewers' knowledge no articles have systematically reviewed the occurrence of ADRs following the use of ADHD medications in the pediatric population.

The objective of this study is to review published empirical studies on the occurrence of adverse drug reactions (ADRs) associated with the use of medications generally licensed for treatment of ADHD symptoms in the pediatric population.

\section{Methods}

\section{Literature search}

A literature search was performed in PubMed, Embase, and PsycINFO $^{\circledR}$ (whole databases without language restriction) using the search terms "atomoxetine" (ATC group N06BA09), "methylphenidate" (ATC group N06BA04), "modafinil”" (ATC group N06BA07), "amphetamine” (ATC group N06BA02), "psychostimulants," and "nonstimulants" combined with any of the following: "adverse drug reaction," "side effect," and "adverse event." Reference lists of identified articles were also screened for additional potentially relevant articles. For further details of the search strategy, please see Appendix 1. Literature searches were updated until September 2011.

\section{Study selection}

Using article titles as the selection basis, the first author retrieved and screened the abstracts to identify studies relevant to the study objective. Potentially relevant articles were retrieved in full text and screened for inclusion. To be considered relevant for this review, articles had to be peer reviewed and report ADRs in children in the age group 0-17 years of age associated with the use of psychostimulants.

Psychostimulants were specified as amphetamine derivates, methylphenidate, and modafinil, and nonstimulants as atomoxetine. Articles reporting ADRs from psychostimulants in mixed populations of children and adults were excluded if age-related ADR occurrence was not specified. Articles were excluded if they did not report data on ADR occurrence that made it possible to calculate rates. Hence, case reports, letters, commentaries, interim analyses, meta-analyses, and review articles were excluded. Further, articles reporting unintended events not classified as ADRs and articles on misuse were excluded, although reference lists of these studies were searched for relevant studies.

\section{Data extraction}

Data from included articles were extracted using a standard form, one for each article. The following information was recorded: authors, publication year, country, study design, dosage, comparator, monitoring period (weeks), size of study population, age and gender of included population, and $\mathrm{ADR}$ reporting rates in percentage. ADR reporting rates were indicated as reported in the original papers. In placebocontrolled studies, information about ADR reporting rates for placebo was also extracted. Information about who had assessed the ADRs, reported ADRs classified as being serious by the respective authors, and funding sources were also recorded. The first author extracted data, while the second author controlled and verified all cases.

\section{Results}

A total of 137 potentially relevant references were identified during the database searches and reference screenings. An overview of the review process and reasons for exclusion are displayed in Figure 1. Sixty-eight studies were excluded after screening abstracts. Sixty-nine studies were retrieved for full text review. Of these studies, four were later excluded as they reported mixed 


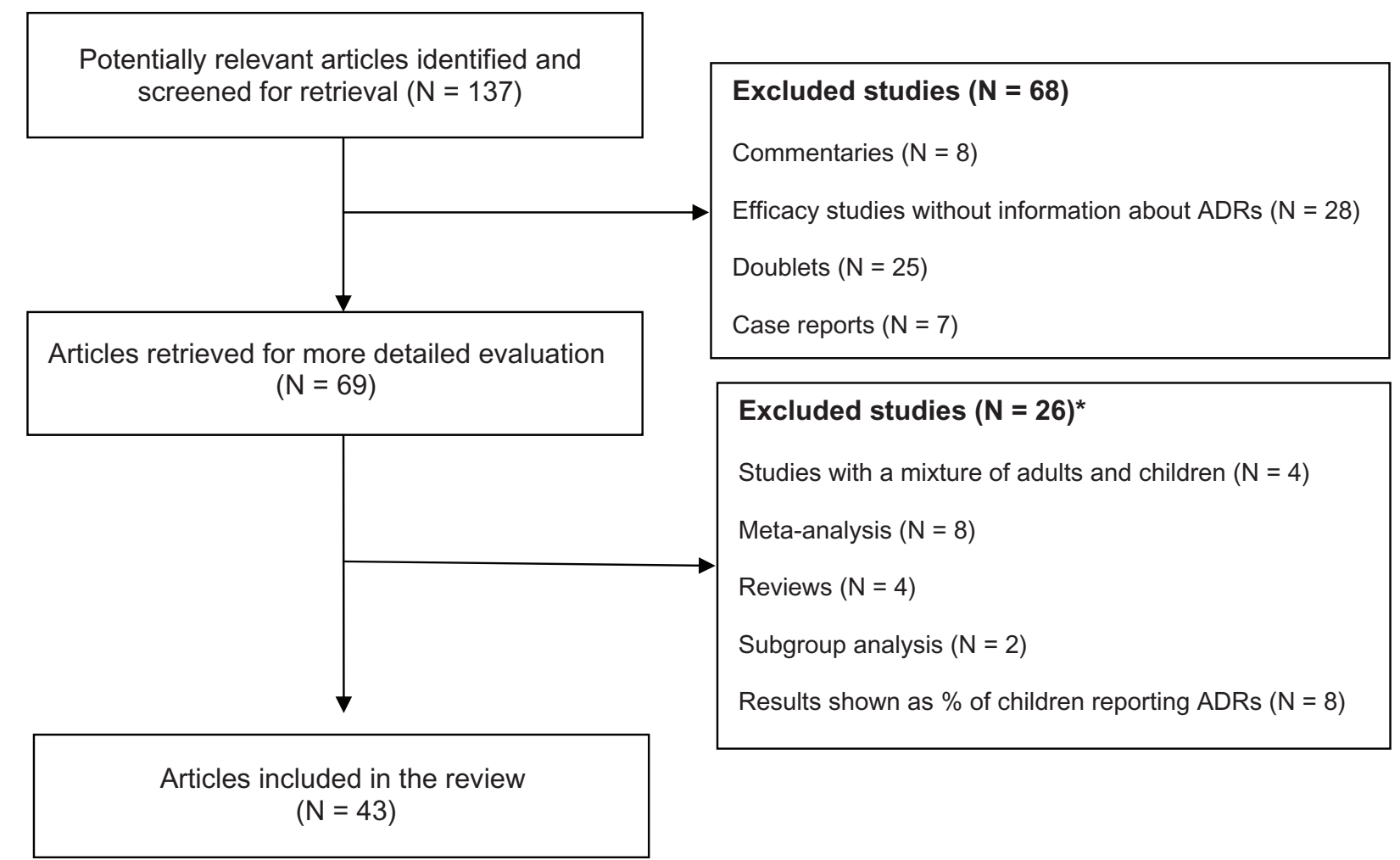

Figure I Decision tree of the review process.

Note: *An overview of excluded studies is shown in Appendix 2.

Abbreviation: ADRs, adverse drug reactions.

data on children and adults that could not be separated. Eight meta-analyses and four reviews of efficacy were excluded as they reported information from studies already included. Also excluded were two studies reporting data from a subgroup analysis of already included studies, and nine studies reporting ADRs as percent of children reporting an ADR.

Eventually 43 articles reporting ADRs from psychostimulants in the pediatric population were included. Table 1 displays an overview of the study characteristics of included articles. The majority of studies were conducted in the United States of America (USA), the remaining in Australia, Canada, Europe, Iran, and Latin America. Atomoxetine studies were published in the period from 2001 to 2009; amphetamine studies from 1997 to 2007; methylphenidate studies from 1997 to 2009; and modafinil studies from 2005 to 2009.

\section{Design and setting}

Information about ADRs was reported in clinical studies using different designs, ie, randomized parallel group studies $(\mathrm{N}=28) ;{ }^{12-15,17-19,22,23,28,31,32,34-36,40,42-47,48-54}$ randomized crossover studies $(\mathrm{N}=6) ;{ }^{16,23,25,27,30,39}$ and open-label designs $(\mathrm{N}=9) .{ }^{20,26,29,33,37,38,41,50}$ The majority of studies were conducted in naturalistic settings at home and at school
$(\mathrm{N}=38) ;^{12,13,15-22,24,26,28-30,31-43,44-54}$ five articles reported ADRs from children participating in laboratory school protocols, ${ }^{14,23,25,27,30}$ in which classroom sessions were organized in cycles to include 12 hours of observation. This design consisted of daily schedules of alternating classroom, meals/snacks, recess, and research activities scheduled at specific times during the day. The largest number of studies $(\mathrm{N}=21)^{31-47}$ concerned atomoxetine; followed by methylphenidate $\left(\mathrm{N}=14 ; ;^{17-30}\right.$ modafinil $(\mathrm{N}=7) ; 48-54$ and amphetamine $(\mathrm{N}=5){ }^{12-16}$

\section{Dosage and comparator}

The tested dosages varied from 10 to $70 \mathrm{mg}$ /day in amphetamine studies; from 5 to $72 \mathrm{mg}$ /day in methylphenidate studies; from 10 to $90 \mathrm{mg}$ /day in atomoxetine studies; and from 100 to $425 \mathrm{mg}$ /day in modafinil studies. Placebo was used as a comparator drug in the majority of studies $(\mathrm{N}=28)$, while an active comparator, was administered in nine studies. Seven open-label studies did not include a control group.

\section{Treatment period}

Treatment duration varied from 1 to 32 weeks across studies. Treatment duration varied from 2 to 4 weeks in amphetamine 
Table I Characteristics of included studies by country, design, study population, and funding

\begin{tabular}{|c|c|c|c|c|c|}
\hline $\begin{array}{l}\text { Studies } \\
\text { (chronological order) }\end{array}$ & Country & Design & Setting & $\begin{array}{l}\text { Dosage } \\
\text { (mg/day) }\end{array}$ & Comparator \\
\hline \multicolumn{6}{|l|}{ Amphetamine } \\
\hline Biederman et a $\left.\right|^{12}$ & USA & $\mathrm{R}$ parallel & Naturalistic & $30-70$ & Placebo \\
\hline Spencer et $\mathrm{al}^{13}$ & USA & $\mathrm{R}$ parallel & Naturalistic & $10-40$ & Placebo \\
\hline Wigal et al ${ }^{14}$ & USA & $\mathrm{R}$ parallel & Laboratory & $10-30$ & Atomoxetine \\
\hline Biederman et al ${ }^{15}$ & USA & $\mathrm{R}$ parallel & Naturalistic & $10-30$ & Placebo \\
\hline Efron et al ${ }^{16}$ & $\mathrm{AU}$ & $\mathrm{R}$ crossover & Naturalistic & $0.15 \mathrm{mg} / \mathrm{kg}$ & $\mathrm{MPH}$ \\
\hline \multicolumn{6}{|l|}{ Total/Range } \\
\hline \multicolumn{6}{|l|}{ Methylphenidate } \\
\hline Arabgol et al ${ }^{17}$ & IR & R parallel & Naturalistic & $20-50$ & Reboxetine \\
\hline Maayan et a $2^{20}$ & USA & Open label & Naturalistic & $10-30$ & NR \\
\hline Amiri et $\mathrm{al}^{49}$ & IR & R parallel & Naturalistic & $20-30$ & Modafinil \\
\hline Findling et $\mathrm{a}^{18}$ & USA & $\mathrm{R}$ parallel & Naturalistic & $10-54$ & Placebo \\
\hline Newcorn et al ${ }^{19}$ & USA & $\mathrm{R}$ parallel & Naturalistic & $18-54$ & Atomoxetine \\
\hline Findling et $\mathrm{al}^{21}$ & Various & $\mathrm{R}$ parallel & Naturalistic & $10-60$ & Placebo \\
\hline Greenhill et al ${ }^{22}$ & USA & $\mathrm{R}$ parallel & Naturalistic & $5-30$ & Placebo \\
\hline McGough et $\mathrm{a}^{23}$ & USA & $\mathrm{R}$ crossover & Laboratory & $10-27$ & Placebo \\
\hline Gau et $\mathrm{al}^{24}$ & TW & R open label & Naturalistic & $10-40$ & None \\
\hline Silva et $\mathrm{al}^{25}$ & USA & $\mathrm{R}$ crossover & Laboratory & $20-40$ & Placebo \\
\hline Kemner et $\mathrm{al}^{26}$ & USA & R open label & Naturalistic & $18-72$ & Atomoxetine \\
\hline Swanson et $\mathrm{al}^{27}$ & USA & $\mathrm{R}$ crossover & Laboratory & $18-60$ & Placebo \\
\hline Biederman et a ${ }^{28}$ & USA/CA & R parallel & Naturalistic & $10-40$ & Placebo \\
\hline Kratochvil et a ${ }^{29}$ & USA/CA & $\mathrm{R}$ open label & Naturalistic & $5-60$ & None \\
\hline Pelham et al ${ }^{30}$ & USA & $\mathrm{R}$ crossover & Lab/Nat & $5-54$ & Placebo \\
\hline Efron et al ${ }^{16}$ & $\mathrm{AU}$ & $\mathrm{R}$ crossover & Naturalistic & $0.3 \mathrm{mg} / \mathrm{kg}$ & Amphetamine \\
\hline \multicolumn{6}{|l|}{ Total/Range } \\
\hline \multicolumn{6}{|l|}{ Atomoxetine } \\
\hline Svanborg et $\mathrm{al}^{3 \mid}$ & SE & R parallel & Naturalistic & 80 & Placebo \\
\hline Block et a ${ }^{32}$ & USA & $\mathrm{R}$ parallel & Naturalistic & $0.47-1.81 \mathrm{mg} / \mathrm{kg}$ & Placebo \\
\hline Tamayo et $\mathrm{al}^{33}$ & Various & Open label & Naturalistic & $35-120$ & None \\
\hline Newcorn et al ${ }^{19}$ & USA & R parallel & Naturalistic & $0.8-1.8 \mathrm{mg} / \mathrm{kg}$ & MPH/Placebo \\
\hline Bangs et al ${ }^{14}$ & US & R parallel & Naturalistic & $1.2-1.8 \mathrm{mg} / \mathrm{kg}$ & Placebo \\
\hline Geller et $\mathrm{a}^{35}$ & USA & $\mathrm{R}$ parallel & Naturalistic & $0.8-1.8 \mathrm{mg} / \mathrm{kg}$ & Placebo \\
\hline Gau et $\mathrm{al}^{36}$ & TW & $\mathrm{R}$ parallel & Naturalistic & $16-99$ & Placebo \\
\hline Kratochvil et a ${ }^{137}$ & USA & Open label & Naturalistic & $0.5-1.8 \mathrm{mg} / \mathrm{kg}$ & None \\
\hline Prasad et $\mathrm{al}^{38}$ & UK & R open label & Naturalistic & $0.5-1.8 \mathrm{mg} / \mathrm{kg}$ & SCT \\
\hline Arnold et al ${ }^{39}$ & USA & $\mathrm{R}$ cross over & Naturalistic & $2.5-40$ & Placebo \\
\hline Newcorn et al ${ }^{40}$ & USA & R parallel & Naturalistic & $1.2-1.8 \mathrm{mg} / \mathrm{kg}$ & None \\
\hline Wigal et al ${ }^{14}$ & USA & R parallel & Laboratory & $10-60$ & Amphetamine \\
\hline Allen et al ${ }^{42}$ & USA & R parallel & Naturalistic & $0.5-1.5 \mathrm{mg} / \mathrm{kg}$ & Placebo \\
\hline Kemner et $\mathrm{al}^{26}$ & USA & $\mathrm{R}$ open label & Naturalistic & $10-80$ & $\mathrm{MPH}$ \\
\hline Escobar et $\mathrm{al}^{41}$ & ES & Open label & Naturalistic & $0.5-1.8 \mathrm{mg} / \mathrm{kg}$ & None \\
\hline Kelsey et $\mathrm{al}^{43}$ & USA & R parallel & Naturalistic & $0.8-1.2 \mathrm{mg} / \mathrm{kg}$ & Placebo \\
\hline Biederman et a ${ }^{44}$ & USA & R parallel & Naturalistic & $2 \mathrm{mg} / \mathrm{kg}$ & Placebo \\
\hline Michelson et al ${ }^{45}$ & USA & $\mathrm{R}$ parallel & Naturalistic & $0.5-1.0 \mathrm{mg} / \mathrm{kg}$ & Placebo \\
\hline Kratochvil et a ${ }^{29}$ & USA/CA & $\mathrm{R}$ open label & Naturalistic & $0.2-2.0 \mathrm{mg} / \mathrm{kg}$ & None \\
\hline Spencer et $\mathrm{a}^{46}$ & USA & R parallel & Naturalistic & 90 & Placebo \\
\hline Michelson et al ${ }^{47}$ & USA & $\mathrm{R}$ parallel & Naturalistic & $0.5-1.8 \mathrm{mg} / \mathrm{kg}$ & Placebo \\
\hline \multicolumn{6}{|l|}{ Total/Range } \\
\hline \multicolumn{6}{|l|}{ Modafinil } \\
\hline Kahbazi et $\mathrm{al}^{48}$ & IR & $\mathrm{R}$ parallel & Naturalistic & $200-300$ & Placebo \\
\hline Amiri et al ${ }^{49}$ & IR & $\mathrm{R}$ parallel & Naturalistic & $200-300$ & $\mathrm{MPH}$ \\
\hline Boellner et $\mathrm{al}^{50}$ & USA & Open label & Naturalistic & $100-400$ & None \\
\hline Wigal et $\mathrm{al}^{51}$ & USA & R parallel & Naturalistic & $170-425$ & Placebo \\
\hline Biederman et $\mathrm{al}^{52}$ & USA & R parallel & Naturalistic & $300-400$ & Placebo \\
\hline Greenhill et $\mathrm{al}^{53}$ & USA & $\mathrm{R}$ parallel & Naturalistic & $170-425$ & Placebo \\
\hline Biederman et a $\left.\right|^{54}$ & USA & $\mathrm{R}$ parallel & Naturalistic & $170-425$ & Placebo \\
\hline \multicolumn{6}{|l|}{ Total/Range } \\
\hline Total all studies & & & & & \\
\hline
\end{tabular}




\begin{tabular}{|c|c|c|c|c|c|c|}
\hline $\begin{array}{l}\text { Treatment } \\
\text { weeks }(\mathbf{N})\end{array}$ & $\begin{array}{l}\text { Patients } \\
\text { included (N) }\end{array}$ & $\begin{array}{l}\text { Patients } \\
\text { completed }(\mathrm{N})\end{array}$ & Age (y) & $\%$ Male & $\begin{array}{l}\text { Type of } \\
\text { assessor }\end{array}$ & Funding \\
\hline 4 & 290 & 218 & $6-12$ & 69 & Parent & Industry \\
\hline 4 & 335 & 308 & $6-17$ & 69 & Parent & Industry \\
\hline 3 & 102 & 93 & $6-12$ & 75 & Parent & Industry \\
\hline 3 & 374 & 336 & $6-12$ & 80 & Parent & Industry \\
\hline 2 & 125 & 121 & $5-15$ & 91 & Teacher/Parent & Nonindustry \\
\hline $2-4$ & 1226 & 1076 & $5-17$ & 69-91 & & \\
\hline 6 & 16 & 12 & $7-16$ & 66 & Teacher/Parent & Nonindustry \\
\hline 4 & 14 & 11 & $4-5$ & 82 & Self & NR \\
\hline 6 & 32 & 30 & $6-15$ & 80 & Teacher/Parent & Nonindustry \\
\hline 7 & 189 & 137 & $6-12$ & 65 & Self & Industry \\
\hline 6 & 220 & 180 & $6-16$ & 71 & Parent & Industry \\
\hline 3 & 272 & 240 & $6-12$ & 80 & Self & Industry \\
\hline 7 & 53 & 48 & $6-17$ & 59 & Self & Industry \\
\hline 5 & 42 & 41 & $6-12$ & 73 & Self & Industry \\
\hline 4 & 64 & 64 & $6-15$ & 91 & Self & Industry \\
\hline$<1$ & 54 & 53 & $6-12$ & 70 & Parent & Industry \\
\hline 3 & 891 & 850 & $6-12$ & 74 & Parent & Industry \\
\hline NR & 184 & $|8|$ & $6-12$ & 74 & Self/Parent & Industry \\
\hline 2 & 65 & 61 & $6-14$ & 80 & Parent & Industry \\
\hline 10 & 44 & 25 & $7-15$ & 100 & Parent & Industry \\
\hline 3 & 70 & 68 & $6-12$ & NR & Teacher/Parent & Industry \\
\hline 2 & 125 & 121 & $5-15$ & 91 & Teacher/Parent & Nonindustry \\
\hline $1-10$ & 2303 & 2092 & $4-17$ & $59-100$ & & \\
\hline 10 & 49 & 49 & $7-15$ & 80 & Self & Industry \\
\hline 6 & 288 & 140 & $6-12$ & 73 & Parent & Industry \\
\hline $\mid 0-11$ & 1198 & 947 & $6-17$ & 76 & Self & Industry \\
\hline 6 & 222 & 186 & $6-16$ & 78 & Parent & Industry \\
\hline 9 & 72 & 71 & $12-17$ & 72 & Self & Industry \\
\hline 12 & 87 & 66 & $8-17$ & 62 & Self & Industry \\
\hline 6 & 72 & 72 & $6-16$ & 90 & Parent & Industry \\
\hline 8 & 22 & 20 & $5-6$ & 86 & Parent & Industry \\
\hline 10 & 104 & 78 & $7-15$ & 89 & Self & Industry \\
\hline 12 & 16 & 15 & $5-15$ & 75 & Self & Industry \\
\hline 32 & 229 & 160 & $6-16$ & 72 & Parent & Industry \\
\hline 3 & 101 & 97 & $6-12$ & 76 & Parent & Industry \\
\hline 18 & 76 & 74 & $7-17$ & 92 & Self & Industry \\
\hline 3 & 499 & 473 & $6-12$ & 74 & Parent & Industry \\
\hline 10 & 36 & 36 & $6-15$ & 89 & Parent & Industry \\
\hline 8 & 133 & 107 & $6-12$ & 71 & Parent & Industry \\
\hline 9 & 31 & 31 & $7-13$ & 0 & Self & Industry \\
\hline 6 & 85 & 84 & $6-16$ & 7I & Parent & Industry \\
\hline 10 & 184 & 118 & $7-15$ & 91 & Parent & Industry \\
\hline 12 & 129 & 127 & $7-13$ & 76 & Parent & Industry \\
\hline 8 & 213 & 176 & $8-17$ & 71 & Self & Industry \\
\hline $3-32$ & 3846 & 3127 & $5-17$ & $0-91$ & & \\
\hline 6 & 24 & 23 & $6-15$ & 76 & Teacher/Parent & Nonindustry \\
\hline 6 & 32 & 30 & $6-15$ & 78 & Teacher/Parent & Nonindustry \\
\hline 8 & 220 & 166 & $6-14$ & 72 & Parent & Industry \\
\hline 9 & 423 & 411 & 10.2 & 72 & Parent/Self & Industry \\
\hline 4 & 197 & 175 & $6-14$ & 75 & Parent & Industry \\
\hline 9 & 133 & 100 & $6-16$ & 73 & Parent/Self & Industry \\
\hline 9 & 164 & 97 & $6-17$ & 69 & Parent & Industry \\
\hline \multirow[t]{2}{*}{$4-9$} & 1137 & 949 & $6-17$ & $69-75$ & & \\
\hline & 8512 & 7244 & - & - & & \\
\hline
\end{tabular}

Abbreviations: AU, Australia; CA, Canada; IR, Iran; lab, laboratory; MPH, methylphenidate; nat, naturalistic; NR, not reported; R, randomized; ES, Spain; SCT, standard current therapy; SE, Sweden; TW, Taiwan; USA, United States of America. 
studies; from 1 to 10 weeks in methylphenidate studies; from 3 to 32 weeks in atomoxetine studies; and from 4 to 9 weeks in modafinil studies.

\section{Population}

A total of 8512 children were included in the clinical studies, of which 7244 children completed treatment: amphetamine (1076); methylphenidate (2092); atomoxetine (3127); and modafinil (949). The reasons for noncompletion were many, but lack of efficacy and the appearance of ADRs were the most common. The ages of the included children varied from 4 to 17 years (median 6-12 years), and the share of male patients in the studies varied from 0 to $100 \%$ (median $69 \%$ ).

\section{Type of assessor}

Parents rated information about ADRs in 20 studies, ${ }^{12-15,19,25-28}$, 32,35-36,40-46,48,50,52 54 patients in 15 studies, ${ }^{18,20-24,31,33-35,38-39,42,44,47}$ and a combination of teacher/parent (five studies), ${ }^{16-17,30,48-49}$ and patient/parent (three studies). ${ }^{27,49,51}$ The articles specified only limited information about applied ADR scales and the classification systems used.

\section{Funding source}

In almost all studies the funding source was the manufacturer of the respective medications, and only four studies were publicly funded. Additionally, a large number of the studies were conducted by the same groups of authors who declared conflicts of interest. The majority of the authors received contributions from the pharmaceutical companies producing the medications in return for activities, such as providing scientific advice and making oral and poster presentations at scientific meetings.

\section{ADRs by type and occurrence}

Tables 2-5 display the ADR reporting rates listed in the included studies for each type of psychostimulant. ADRs of similar type and wording were aggregated in a common category in order to clarify data presentation. The aggregated categories were: weight changes (changes in weight, weight decreased, weight increased, decrease in weight); gastrointestinal pain (abdominal pain, upper abdominal pain, gastrointestinal pain); anxiety (anxiety, anxiousness); influenza (influenza, flu syndrome); tics (tics, motor tics, facial tics); blood pressure changes (diastolic blood pressure, changes in blood pressure); sleeping problems (awake during the night, difficulty falling asleep, sleep disturbance, delayed onset of sleep); changes in heart rate (racing heart, changes in heart rate). Thirty-one categories of ADRs were reported for amphetamine derivates (Table 2); 65 categories for methylphenidate formulations (Table 3); 55 categories for atomoxetine (Table 4); and 38 categories for modafinil (Table 5). The following ADRs were most frequently reported for all four psychostimulants: decrease in appetite, gastrointestinal pain, and headache.

\section{ADRs by seriousness}

The majority of reported ADRs were categorized by the authors/investigators as nonserious. Table 6 shows information about the categories of serious ADRs reported in the clinical studies. Serious cases included aggression (amphetamine, methylphenidate); ${ }^{16}$ anxiety (amphetamine) $;{ }^{16}$ emotional disturbances (amphetamine); ${ }^{14,15}$ insomnia

Table 2 Adverse drug reaction reporting rates (\%) for amphetamine derivates by category and study

\begin{tabular}{|c|c|c|c|c|c|c|c|}
\hline Reference number & 12 & 13 & 14 & 15 & 16 & Range & $\begin{array}{l}\text { Placebo } \\
\text { (range) }\end{array}$ \\
\hline \multicolumn{8}{|l|}{ Adverse drug reaction } \\
\hline Accidental injury & - & 5 & - & - & - & 5 & 5 \\
\hline Anorexia & - & 25 & 17 & 22 & - & $17-25$ & 2 \\
\hline Anxiety & - & - & - & - & 68 & 68 & - \\
\hline Appetite decrease & 39 & - & 28 & - & 59 & $28-59$ & $4-5$ \\
\hline Cough & I & - & - & 5 & - & $\mathrm{I}-5$ & 5 \\
\hline Crying & - & - & - & - & 76 & 76 & - \\
\hline Daydreams & - & - & - & - & 62 & 62 & - \\
\hline Dizziness & 5 & - & 6 & - & 32 & $5-32$ & - \\
\hline Dry mouth & 5 & - & - & - & - & 5 & - \\
\hline Emotional disturbance & - & - & - & - & 59 & 59 & - \\
\hline Emotional lability & - & 5 & - & 9 & - & $5-9$ & 2 \\
\hline Fatigue & - & - & 2 & - & - & 2 & - \\
\hline Fingernail biting & - & - & - & - & 40 & 40 & - \\
\hline Gastrointestinal pain & 12 & 11 & 19 & 14 & - & $11-19$ & $5-10$ \\
\hline Headache & 12 & 19 & 15 & 18 & 30 & $12-30$ & $|0-2|$ \\
\hline Insomnia & 19 & 20 & 28 & 17 & - & $17-28$ & $2-8$ \\
\hline Irritability & 10 & - & 7 & - & 82 & $7-82$ & - \\
\hline Nasal congestion & 1 & - & - & - & - & 1 & - \\
\hline Nasopharyngitis & 5 & - & - & - & - & 5 & - \\
\hline Nausea & 6 & - & 7 & 5 & - & $5-7$ & 3 \\
\hline Nervousness & - & 6 & - & 6 & - & 6 & 2 \\
\hline Nightmares & - & - & - & - & 28 & 28 & - \\
\hline Pharyngitis & - & 7 & - & 7 & - & 7 & $5-20$ \\
\hline Sleeping problems & - & - & - & - & 70 & 70 & - \\
\hline Social withdrawal & - & - & - & - & 64 & 64 & - \\
\hline Somnolence & - & - & 5 & - & - & 5 & - \\
\hline Stomachache & - & - & - & - & 40 & 40 & - \\
\hline Tics & - & - & - & - & 26 & 26 & - \\
\hline Unusually happy & - & - & - & - & 26 & 26 & - \\
\hline Vomiting & 9 & - & 5 & 7 & - & $5-9$ & 4 \\
\hline Weight changes & 9 & 8 & 6 & - & - & $6-9$ & $\mathrm{I}$ \\
\hline
\end{tabular}


Table 3 Adverse drug reaction reporting rates (\%) for methylphendiate by category and study

\begin{tabular}{|c|c|c|c|c|c|c|c|c|c|c|c|c|c|c|c|c|c|c|}
\hline Reference number & 16 & 17 & 18 & 19 & 20 & 21 & 22 & 23 & 24 & 25 & 26 & 27 & 28 & 29 & 30 & 49 & Range & $\begin{array}{l}\text { Placebo } \\
\text { (range) }\end{array}$ \\
\hline \multicolumn{19}{|l|}{ Adverse drug reaction } \\
\hline Abnormal behavior & - & - & - & - & - & 3 & - & - & - & - & I & - & - & 5 & - & - & $\mathrm{I}-5$ & 4 \\
\hline Accidental injury & - & - & - & - & - & - & - & - & - & - & - & 3 & - & 13 & 3 & - & $3-13$ & 3 \\
\hline Affect lability & - & - & 3 & - & - & - & 4 & - & - & - & - & - & - & - & - & - & $3-4$ & - \\
\hline Aggression & - & - & - & - & - & - & - & - & - & - & 1 & - & - & - & - & - & I & - \\
\hline Anorexia & - & - & 3 & - & - & 5 & 4 & 3 & - & 8 & - & 3 & 3 & 15 & - & - & $3-15$ & $1-2$ \\
\hline Anxiety & 61 & - & - & - & - & - & - & - & 25 & - & - & - & - & - & - & 5 & $5-61$ & I \\
\hline Appetite decrease & 56 & 31 & 19 & 17 & 28 & 3 & 30 & - & 53 & 9 & 6 & 3 & - & - & - & 31 & $3-56$ & $5-9$ \\
\hline Asthenia & - & - & - & - & - & - & - & - & - & - & - & - & - & 3 & - & - & 3 & - \\
\hline Blood pressure changes & - & - & - & 18 & - & - & - & 3 & - & - & - & - & - & - & - & - & $3-18$ & - \\
\hline Changes in heart rate & - & - & - & 12 & - & - & - & - & - & - & - & - & - & - & - & - & 12 & - \\
\hline Changes in pulse rate & - & - & - & - & - & - & - & - & - & I & - & - & - & - & - & - & 1 & - \\
\hline Chest pain & - & 6 & - & - & - & - & - & - & - & - & - & - & - & - & - & - & 6 & - \\
\hline Cough & - & - & - & 4 & - & 2 & - & - & - & - & - & - & - & 5 & - & - & $2-5$ & 4 \\
\hline Crying & 71 & - & - & - & - & - & - & - & 38 & - & 2 & - & - & - & - & - & $2-7 \mid$ & - \\
\hline Daydreams & 62 & - & - & - & - & - & - & - & 30 & - & - & - & - & - & - & - & $30-62$ & - \\
\hline Depression & - & - & - & - & - & - & - & - & - & - & - & - & - & 5 & - & - & 5 & - \\
\hline Diarrhea & - & - & - & - & - & - & 4 & - & - & - & - & - & - & 3 & 2 & - & $2-4$ & $\mathrm{I}-2$ \\
\hline Dizziness & 30 & 13 & - & - & - & - & - & - & 13 & - & 1 & - & - & - & 2 & - & $1-30$ & 4 \\
\hline Dry mouth & - & - & - & - & - & - & - & - & 24 & - & - & - & - & - & - & 12 & $12-24$ & - \\
\hline Dyspepsia & - & - & - & - & - & - & 8 & - & - & - & - & - & - & 5 & - & - & $5-8$ & - \\
\hline Emotional disturbance & 56 & - & - & - & - & - & - & - & - & - & 1 & - & - & - & - & - & $\mathrm{I}-56$ & - \\
\hline Emotional lability & - & - & - & - & 8 & - & - & - & - & - & - & - & - & 5 & - & 7 & $5-8$ & - \\
\hline Euphoria & - & - & - & - & - & - & - & - & 9 & - & - & - & - & - & - & - & 9 & - \\
\hline Eye redness & - & - & - & - & 4 & - & - & - & - & - & - & - & - & - & - & - & 4 & - \\
\hline Eye twitching & - & - & - & - & 4 & - & - & - & - & - & - & - & - & - & - & - & 4 & - \\
\hline Fatigue & - & - & - & 2 & 4 & - & 4 & - & - & 4 & 1 & - & - & - & - & - & $\mathrm{I}-4$ & 4 \\
\hline Fever & - & - & - & - & 4 & 2 & - & - & - & - & - & - & - & 10 & - & - & $2-10$ & 7 \\
\hline Fingerrnail biting & 45 & - & - & - & - & - & - & - & 22 & - & - & - & - & - & - & - & $22-45$ & - \\
\hline Gastroenteritis & - & - & - & - & - & - & 4 & - & - & - & - & 5 & - & - & - & - & $4-5$ & 4 \\
\hline Gastrointestinal pain & - & - & - & 10 & 12 & 10 & 19 & - & - & 6 & 4 & 4 & - & 18 & 15 & 8 & $4-19$ & $2-13$ \\
\hline Headache & 24 & - & - & 11 & 4 & 16 & 25 & 4 & 28 & 2 & 4 & 3 & 2 & 33 & 14 & 8 & $2-33$ & $3-23$ \\
\hline Hyperkinesia & - & - & - & - & - & - & - & - & - & - & - & - & - & 5 & - & - & 5 & - \\
\hline Increases in ALT/AST & - & - & - & - & - & - & - & - & - & - & - & 5 & - & - & - & - & 5 & - \\
\hline Infection & - & - & - & - & 4 & - & - & - & - & - & - & 2 & - & 8 & - & - & $2-8$ & $\mathrm{I}-4$ \\
\hline Influenza & - & - & - & - & - & - & 4 & - & - & - & - & - & - & 10 & - & - & $4-10$ & - \\
\hline Insomnia & - & 19 & 8 & 27 & - & 4 & 8 & - & 44 & 4 & 7 & 2 & 3 & 18 & - & - & $2-44$ & $3-10$ \\
\hline Irritability & 80 & 6 & - & 6 & 4 & 3 & 4 & - & 16 & - & 1 & 1 & - & - & - & 7 & $\mathrm{I}-80$ & $2-6$ \\
\hline Lymphadenopathy & - & - & - & - & - & - & - & 3 & - & - & - & - & - & - & - & - & 3 & - \\
\hline Mood alteration & - & - & - & - & - & - & - & - & 34 & - & 1 & 7 & - & - & - & - & $\mathrm{I}-34$ & - \\
\hline Nasal congestion & - & - & 3 & - & 4 & - & - & - & - & - & - & - & - & - & - & - & $3-4$ & 1 \\
\hline Nasopharyngitis & - & - & 4 & - & - & 4 & 9 & I & - & - & - & - & - & - & - & - & $1-9$ & $2-7$ \\
\hline Nausea & - & - & 8 & 6 & - & - & 11 & 4 & - & - & 1 & - & - & 5 & - & 5 & $I-I I$ & $2-6$ \\
\hline Nervousness & - & - & - & - & - & - & - & - & - & - & - & 4 & - & 10 & - & - & $4-10$ & - \\
\hline Nightmare & 21 & - & - & - & - & - & - & - & 16 & - & - & - & - & - & - & - & $|6-2|$ & - \\
\hline Otitis media & - & - & - & - & - & - & 4 & - & - & - & - & - & - & - & - & - & 4 & 2 \\
\hline Pain & - & - & - & - & - & - & - & - & - & - & - & - & - & 3 & - & - & 3 & - \\
\hline Pallor & - & 13 & - & - & - & - & - & - & - & - & - & - & - & - & - & - & 13 & - \\
\hline Palpitation & - & - & - & - & - & - & - & - & - & - & - & - & - & 5 & - & - & 5 & - \\
\hline Pharyngeal pain & - & - & - & - & - & - & - & 3 & - & - & - & - & - & - & - & - & 3 & - \\
\hline Pharyngitis & - & - & - & - & - & 3 & - & - & - & - & - & - & - & 8 & 2 & - & $2-8$ & 3 \\
\hline Rash & - & - & - & - & - & - & - & 1 & - & - & - & - & - & 8 & - & - & $1-8$ & 4 \\
\hline Respiratory tract infection & - & - & - & - & - & 3 & 9 & - & - & - & - & - & - & - & 4 & - & $3-9$ & $2-6$ \\
\hline Rhinitis & - & - & - & - & - & - & - & 3 & - & - & - & - & - & 20 & 2 & - & $2-20$ & - \\
\hline Sensitivity & - & - & - & - & 4 & - & - & - & - & - & - & - & - & - & - & - & 4 & - \\
\hline Sleeping problems & 64 & - & - & - & 12 & - & - & - & - & - & - & - & - & - & - & 9 & $9-64$ & - \\
\hline
\end{tabular}


Table 3 (Continued)

\begin{tabular}{|c|c|c|c|c|c|c|c|c|c|c|c|c|c|c|c|c|c|c|}
\hline Reference number & 16 & 17 & 18 & 19 & 20 & 21 & 22 & 23 & 24 & 25 & 26 & 27 & 28 & 29 & 30 & 49 & Range & $\begin{array}{l}\text { Placebo } \\
\text { (range) }\end{array}$ \\
\hline Socially withdrawn & 59 & - & - & - & - & - & - & - & 27 & - & - & - & - & - & - & - & $27-59$ & - \\
\hline Somnolence & - & - & - & 2 & - & - & - & - & - & - & I & - & - & - & - & - & $\mathrm{I}-2$ & - \\
\hline Stomachache & 32 & - & - & - & - & - & 4 & - & 28 & - & - & - & - & - & - & - & $4-32$ & - \\
\hline Tachycardia & - & - & - & - & - & - & - & - & - & - & - & - & - & 5 & - & - & 5 & - \\
\hline Tics & 28 & - & I & - & - & - & - & - & 13 & - & - & - & - & - & - & - & $\mathrm{I}-28$ & 4 \\
\hline Twitching & - & - & - & - & - & - & - & - & - & - & - & - & - & - & 3 & - & 3 & - \\
\hline Unusually happy & 28 & - & - & - & - & - & - & - & - & - & - & - & - & - & - & - & 28 & - \\
\hline Urinary incontinence & - & - & - & - & - & - & - & - & - & - & - & - & - & - & I & - & I & 3 \\
\hline Vomiting & - & - & 10 & 4 & 4 & 3 & 4 & - & - & - & I & I & - & - & 3 & - & $1-10$ & $2-5$ \\
\hline Weight changes & - & - & 8 & I & - & - & - & - & - & - & - & - & - & 5 & - & 8 & $1-8$ & 4 \\
\hline
\end{tabular}

Abbreviation: AST/ALT, aspartate aminotransferase/alanine aminotransferase.

(amphetamine, modafinil); ${ }^{13,15,37}$ and attempted suicide (amphetamine). ${ }^{13}$

\section{Discussion}

This is the first study to systematically review the empirical literature on the occurrence of ADRs reported for ADHD medications in the pediatric population. Information about ADRs from psychostimulants and the nonstimulant atomoxetine was reported in clinical studies of short duration, primarily conducted in 6- to 12-year-old boys, and particularly in the USA. The most frequently reported ADRs were decrease in appetite, gastrointestinal pain, and headache. A large number of studies were conducted by the same groups of authors and sponsored by the pharmaceutical companies manufacturing the respective medications.

\section{Design and setting}

Although the review process found a large number of small clinical trials exploring the efficacy of ADHD medications in the pediatic population, only a minor share of these studies reported information about ADRs. The studies included in this article were similar in design and setting, treatment duration, as well as number, age, and gender of included patients. The reliability of the studies may be questioned as the number of reported ADRs varied widely for identical and similar study designs. Further exploration of these questions would require access to the original study material. Large variations in ADR reporting rates were observed between studies and therapeutic groups, and similar types of ADRs were reported for the individual ADHD medications. It is puzzling that large numbers of specific ADRs are reported in some studies, but few if any in others. These findings question the relevance of the many small clinical trials conducted on the medications, particularly atomoxetine and methylphenidate, as they are not designed to measure long-term efficacy and safety. ${ }^{55}$ Almost all of these clinical trials were sponsored by the pharmaceutical companies producing the subject medications, and therefore, the current reviewers encourage these companies to make information about the ADRs reported in said clinical trials accessible to the public.

\section{Seriousness of reported ADRs}

Only a small number of serious ADRs were reported. However, in several of the included studies a large number of children withdrew due to experiencing ADRs, and therefore, the actual number of serious ADRs occurring from the use of ADHD medications might be higher, and some types of ADRs may not have been reported. Information about ADR incidence in the monitored population was only reported if the incidence was above $2 \%$ and/or $5 \%$; consequently, information about rarely occurring ADRs is not included. Another issue is that information about definitions and scales to define and evaluate events occurring during the clinical trials is not reported in the articles, thus making it impossible to react to this information. Therefore, the regulatory agencies are encouraged to allow access to the original clinical protocols, so that all information reported for ADHD medications can be made public. A previous study has shown that there are large discrepancies between the data reported in clinical trial protocols and data published in scientific journals. ${ }^{56}$

\section{Long-term safety aspects of psychostimulant use}

Psychostimulants and other ADHD medications are prescribed for long-term treatment in large populations and 


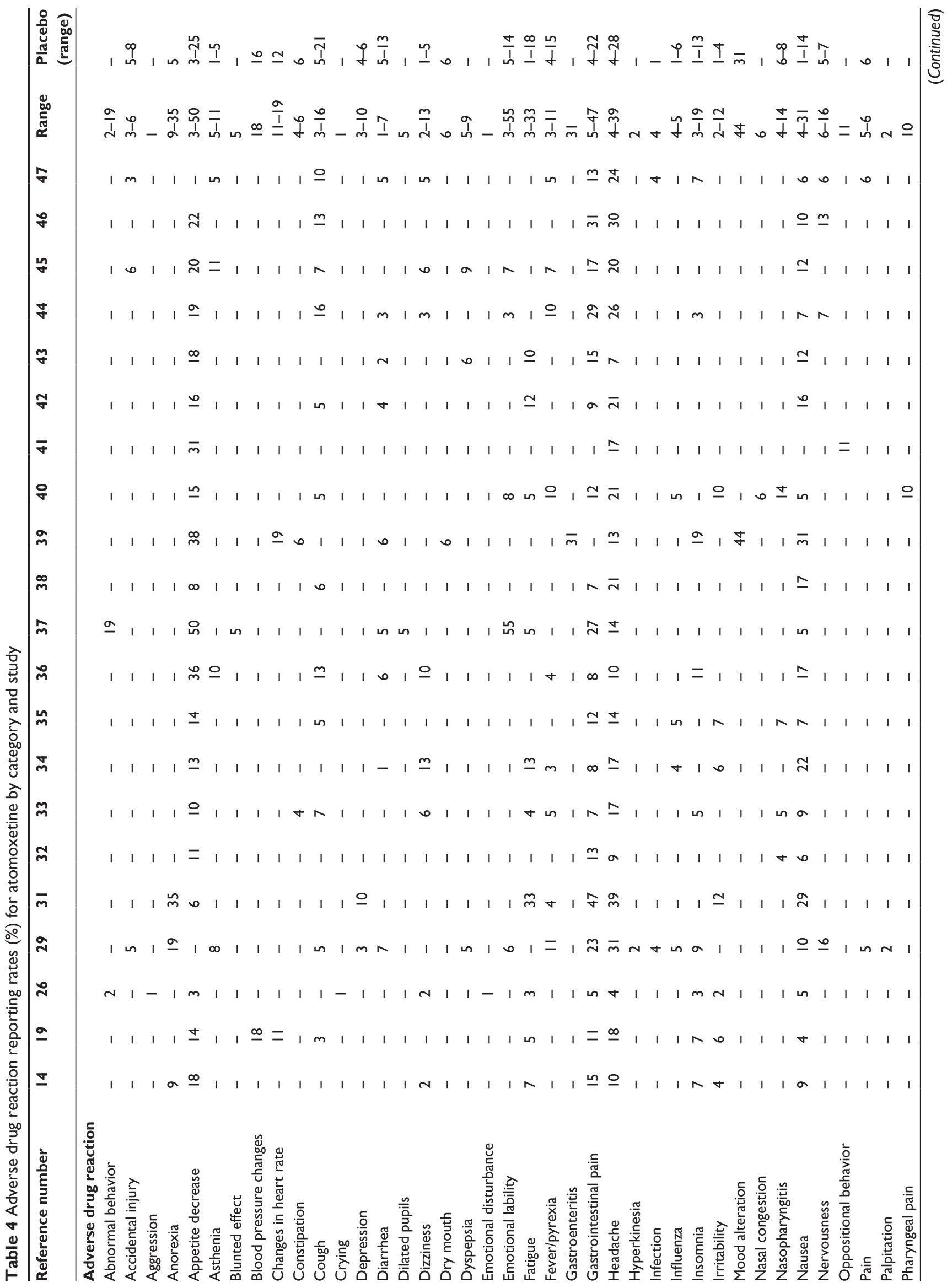




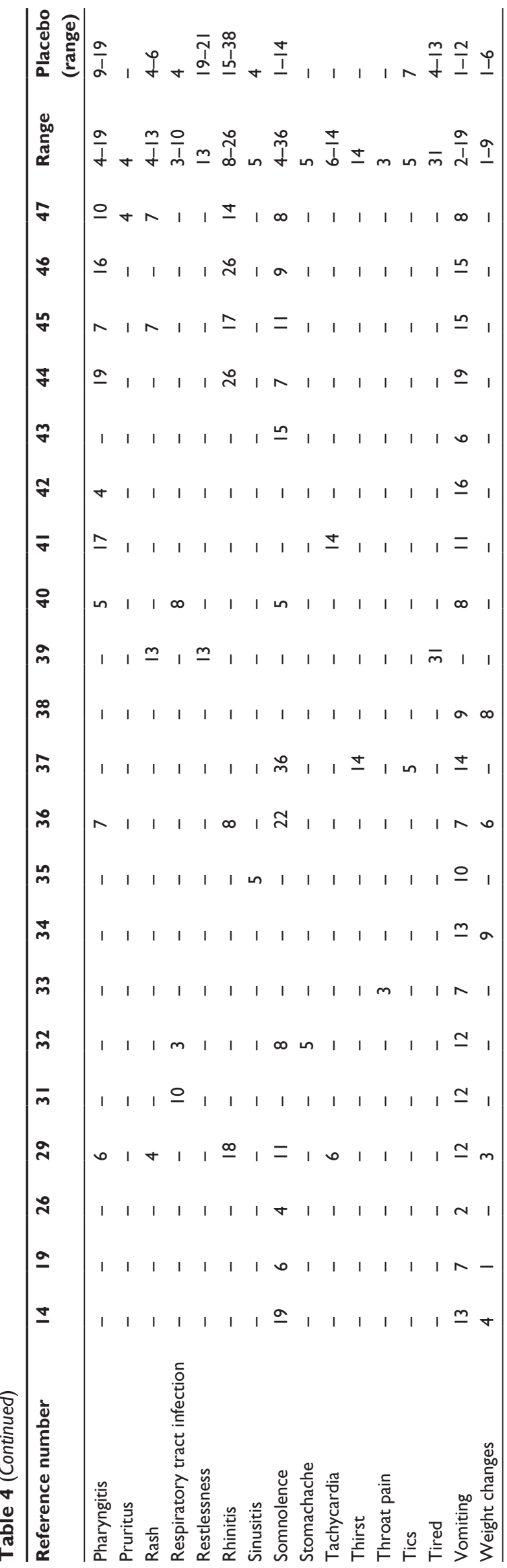

there is a need for long-term efficacy and safety studies. ${ }^{1}$ The lack of sufficient knowledge about ADRs at the point of licensing of new medicines makes spontaneous ADR reporting an important source of information about medicine safety. ${ }^{57}$ As clinical trials in the pediatric population are limited, clinicians and health authorities must rely on spontaneous reports as the main source of information about previously unknown ADRs. ${ }^{57}$ However, the current review did not find any studies about ADRs from the use of psychostimulants reported to any national ADR databases. Systematic analyses of ADRs reported to national databases are necessary, as these databases constitute a critical (and underestimated) source of important data, especially information about new, serious, and rarely occurring ADRs. Further studies of data from large databases, ie, the World Health Organization/Uppsala Monitoring Centre VigiBase $^{\text {TM }}$ (Uppsala, Sweden) or the European Medicines Agency EudraVigilance (London, United Kingdom [UK]) databases, are recommended in order to increase knowledge about ADRs from the use of ADHD medications.

\section{Strengths and limitations of this review}

The included studies were conducted over a period of approximately 20 years in different countries, with a great deal of inconsistency in observing and classifying the type and seriousness of reported ADRs. Information about the seriousness of the reported ADRs was extracted from the included studies, and it was not possible for the review to evaluate these ratings, nor to estimate ADRs in terms of effect sizes, as the review did not have access to the original data material. A major limitation of this study is that it is unknown to what extent the causality of these ADRs can be confirmed, and this has implications for the interpretation of the findings in the review. A large number of published clinical studies were not included in this review because these articles did not report information about ADRs, despite the fact that pharmaceutical companies had a legal obligation to monitor ADRs in clinical trials, and therefore, these data must exist. As the clinical trials were mainly sponsored by the pharmaceutical companies that produce the medications, these companies are urged to make these data accessible to the public.

\section{Conclusion}

Reported ADRs from the use of psychostimulants in the pediatric population were generally found in clinical trials of short duration. Since ADHD medications are prescribed for long-term treatment there is a need for long-term safety 


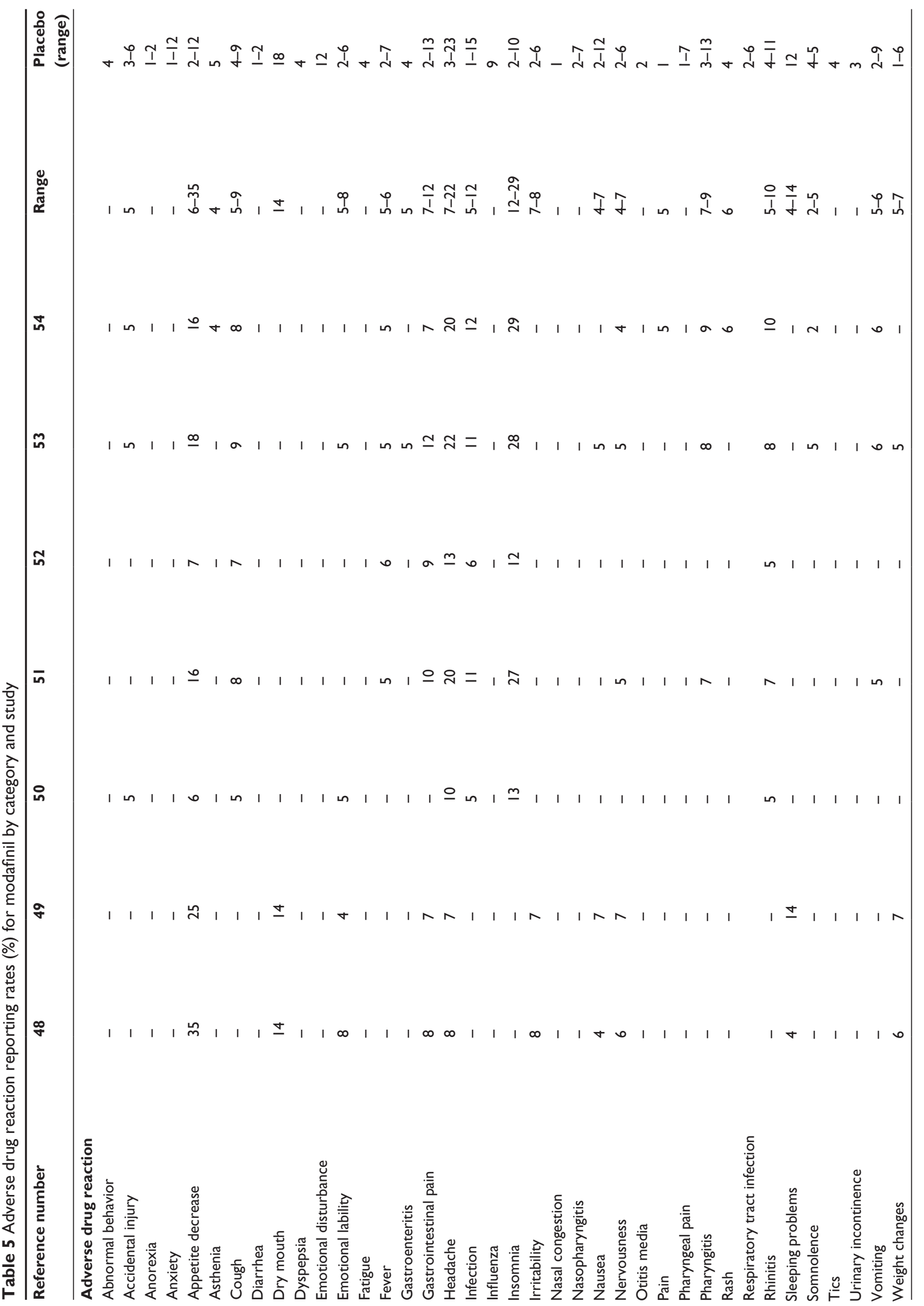


Table 6 Serious ADRs reported for ADHD medications in identified studies

\begin{tabular}{|c|c|c|}
\hline $\begin{array}{l}\text { Medication } \\
\text { (alphabetically) }\end{array}$ & Reference & Adverse drug reaction(s) \\
\hline \multirow[t]{14}{*}{ Amphetamine } & Spencer et $\mathrm{al}^{13}$ & Arthrosis \\
\hline & & Hyperkinesia \\
\hline & & Insomnia \\
\hline & & Nervousness \\
\hline & & Pharyngitis \\
\hline & & Suicide attempt \\
\hline & Wigal et al ${ }^{14}$ & Emotional disturbance \\
\hline & & Headache \\
\hline & Biedermann et al $^{15}$ & Anorexia \\
\hline & & Emotional lability \\
\hline & & Insomnia \\
\hline & Efron et $\mathrm{al}^{16}$ & Agitation \\
\hline & & Aggression \\
\hline & & Anxiety \\
\hline \multirow[t]{6}{*}{ Methylphenidate } & Efron et $\mathrm{al}^{16}$ & Aggression \\
\hline & & Headache \\
\hline & & Tearful \\
\hline & Greenhill et $\mathrm{al}^{22}$ & Hypersomnia \\
\hline & Kemner et $\mathrm{al}^{26}$ & Mania \\
\hline & Biederman et $\mathrm{al}^{28}$ & Depression \\
\hline \multirow[t]{2}{*}{ Atomoxetine } & Wigal et al $^{14}$ & Upper abdominal pain \\
\hline & Arnold et al ${ }^{39}$ & Aggression \\
\hline \multirow[t]{10}{*}{ Modafinil } & Boellner et $\mathrm{al}^{50}$ & Insomnia \\
\hline & Biederman et $\mathrm{al}^{52}$ & Insomnia \\
\hline & Wigal et $\mathrm{al}^{51}$ & Asthma \\
\hline & & Dehydration \\
\hline & & Duodenitis \\
\hline & & Erythema multiforme \\
\hline & & Hypertonia \\
\hline & & Influenza syndrome \\
\hline & & Peptic ulcer \\
\hline & & Stevens-Johnson syndrome \\
\hline
\end{tabular}

Abbreviations: ADRs, adverse drug reactions; ADHD, attention deficit hyperactivity disorder.

studies. Considering the widespread and increasing use of these medications in children, greater care must be taken when prescribing these medications for long-term use. Further studies of spontaneous reports submitted to national and international databases are recommended in order to increase knowledge about ADRs from the use of psychostimulants in the pediatric population. Pharmaceutical companies should make all information about ADRs reported for ADHD medications accessible to the public. Additionally, the impact of the link between researchers and the manufacturers of the medications needs to be studied.

\section{Acknowledgments}

We wish to thank Ditte Sloth-Lisbjerg, MSc (Pharm.), for assistance with parts of the literature search and data extraction.

\section{Authors' contributions}

LA and EHH designed the study, analyzed the data, and wrote the final draft of the manuscript. LA conducted the literature search and data extraction. EHH checked all data extractions. Both authors read and approved the final version of the manuscript.

\section{Disclosure}

The authors report no conflicts of interest in this work.

\section{References}

1. American Academy of Pediatrics. Clinical practice guideline: treatment of the school-aged child with attention-deficit/hyperactivity disorder. Pediatrics. 2001;108(4):1033-1044.

2. European Medicines Agency. Meeting Highlights From the Committee for Medicinal Products for Human Use, 16-19 July 2007. Doc. Ref. EMEA/431407/2007. London: European Medicines Agency; 2007. Available from: http://www.ema.europa.eu/docs/en_GB/document_library/ Press_release/2009/12/WC500017068.pdf. Accessed October 31, 2011.

3. Novartis Pharmaceuticals Corporation, United States Food and Drug Administration. Ritalin ${ }^{\circledR}$, Ritalin-SR ${ }^{\circledR}$. East Hanover, New Jersey: Novartis Pharmaceuticals Corporation; 2007. Available from: http:// www.accessdata.fda.gov/drugsatfda_docs/label/2007/010187s069,01 8029s040,021284s011lbl.pdf. Accessed October 31, 2011.

4. Mosholder AD. Overview of ADHD and its pharmacotherapy. Gaithersburg, MD: United States Food and Drug Administration, Drug Safety and Risk Management Advisory Committee; February 9, 2006. Available from: http://www.fda.gov/ohrms/dockets/ac/06/slides/20064202S1_01_FDA-mosholder.ppt. Accessed July 18, 2011.

5. Nutt DJ, Fone K, Asherson P, et al. Evidence-based guidelines for management of attention-deficit/hyperactivity disorder in adolescents and in adults: recommendations from the British Association for Psychopharmacology. J Psychopharmacol. 2007;21(1):10-41.

6. Trip AM, Visser ST, Kalverdijk LJ. Large increase of the use of psychostimulants among youth in The Netherlands between 1996 and 2006. Br J Clin Pharmacol. 2009;67(4):466-468.

7. Schubert I, Köster I, Lehmkuhl G. The changing prevalence of attentiondeficit/hyperactivity disorder and methylphenidate prescriptions: a study of data from a random sample of insurees of the AOK Health Insurance Company in the German State of Hesse, 2000-2007. Dtsch Arztebl Int. 2010;107(36):615-621.

8. Asheim H, Nilsen KB, Johansen K, et al. Forskvivning av sentralstimulerende legemidler ved $\mathrm{AD} / \mathrm{HD}$ i Nordland [Prescribing of stimulants for ADHD in Nordland County]. Tidsskr Nor Laegeforen. 2007;127: 2360-2362. Article in Norwegian.

9. Zuvekas SH, Vitiello B, Norquist GS. Recent trends in the stimulant medication use among US children. Am J Psychiatry. 2006;163(4):579-585.

10. Bloch MH, Panza KE, Landeros-Weisenberger A, et al. Meta-analysis: treatment of attention-deficit/hyperactivity disorder in children with comorbid tic disorders. J Am Acad Child Adolesc Psychiatry. 2009;48(9):884-893.

11. Aagaard L, Thirstrup S, Hansen EH. Opening the white boxes: the licensing documentation of efficacy and safety of psychotropic medicines for children. Pharmacoepidemiol Drug Saf. 2009;18(5):401-411.

12. Biederman J, Krishnan S, Zhang Y, et al. Efficacy and tolerability of lisdexamfetamine dimesylate (NRP-104) in children with attention-deficit/ hyperactivity disorder: a phase III, multicenter, randomized, double-blind, forced-dose, parallel-group study. Clin Ther. 2007;29(3):450-463.

13. Spencer TJ, Abikoff HB, Connor DF, et al. Efficacy and safety of mixed amphetamine salts extended release (Adderall XR) in the management of oppositional defiant disorder with or without comorbid attentiondeficit/hyperactivity disorder in school-aged children and adolescents: a 4-week, multicenter, randomized, double-blind, parallel-group, placebocontrolled, forced-dose-escalation study. Clin Ther. 2006;28:402-418. 
14. Wigal SB, McGough JJ, McCracken et al. A laboratory school comparison of mixed amphetamine salts extended release (Adderall XR) and atomoxetine (Strattera) in school-aged children with attention deficit/hyperactivity disorder. JAtten Disord. 2005;9(1): 275-289.

15. Biederman J, Lopez FA, Boellner SW, et al. A randomized, doubleblind, placebo-controlled, parallel-group study of SLI381 (Adderall $\mathrm{XR}$ ) in children with attention-deficit/hyperactivity disorder. Pediatrics. 2002;110(2):258-266.

16. Efron D, Jarman F, Barker M. Side effects of methylphenidate and dexamphetamine in children with attention deficit hyperactivity disorder: a double-blind, crossover trial. Pediatrics. 1997;100(4): 662-666.

17. Arabgol F, Panaghi L, Hebrani P. Reboxetine versus methylphenidate in treatment of children and adolescents with attention deficit-hyperactivity disorder. Eur Child Adolesc Psychiatry. 2009;18(1):53-59.

18. Findling RL, Bukstein OG, Melmed RD, et al. A randomized, doubleblind, placebo-controlled, parallel-group study of methylphenidate transdermal system in pediatric patients with attention-deficit/hyperactivity disorder. J Clin Psychiatry. 2008;69(1):149-159.

19. Newcorn JH, Kratochvil CJ, Allen AJ, et al. Atomoxetine and osmotically released methylphenidate for the treatment of attention deficit hyperactivity disorder: acute comparison and differential response. Am J Psychiatry. 2008;165(6):721-730.

20. Maayan L, Paykina N, Fried J, et al. The open-label treatment of attention-deficit/hyperactivity disorder in 4- and 5-year-old children with beaded methylphenidate. J Child Adolesc Psychopharmacol. 2009;19(2):147-153.

21. Findling R, Quinn D, Hatch SJ, et al. Comparison of the clinical efficacy of twice-daily Ritalin and once-daily Equasym XL with placebo in children with Attention-Deficit/Hyperactivity Disorder. Eur Child Adolesc Psychiatry. 2006;15(8):450-459.

22. Greenhill LL, Muniz R, Ball RR, et al. Efficacy and safety of dexmethylphenidate extended-release capsules in children with attentiondeficit/hyperactivity disorder. J Am Acad Child Adolesc Psychiatry. 2006;45(7):817-823.

23. McGough JJ, Wigal SB, Abikoff $\mathrm{H}$, et al. A randomized, double-blind, placebo-controlled, laboratory classroom assessment of methylphenidate transdermal system in children with ADHD. J Atten Disord. 2006;9(3):476-485.

24. Gau SS, Shen HY, Soong WT, et al. An open-label, randomized, activecontrolled equivalent trial of osmotic release oral system methylphenidate in children with attention-deficit/hyperactivity disorder in Taiwan. J Child Adolesc Psychopharmacol. 2006;16(4):441-455.

25. Silva RR, Muniz R, Pestreich L, et al. Efficacy and duration of effect of extended-release dexmethylphenidate versus placebo in schoolchildren with attention-deficit/hyperactivity disorder. $J$ Child Adolesc Psychopharmacol. 2006;16(3):293-251.

26. Kemner JE, Starr HL, Ciccone PE, et al. Outcomes of OROS methylphenidate compared with atomoxetine in children with ADHD: a multicenter, randomized prospective study. Adv Ther. 2005;22(5):498-512.

27. Swanson JM, Wigal SB, Wigal T, et al. A comparison of once-daily extended-release methylphenidate formulations in children with attention-deficit/hyperactivity disorder in the laboratory school (the Comacs Study). Pediatrics. 2004;113(3 Pt 1):e206-e216.

28. Biederman J, Quinn D, Weiss M, et al. Efficacy and safety of Ritalin LA, a new, once daily, extended-release dosage form of methylphenidate, in children with attention deficit hyperactivity disorder. Paediatr Drugs. 2003;5(12):833-841.

29. Kratochvil CJ, Heiligenstein JH, Dittmann R, et al. Atomoxetine and methylphenidate treatment in children with ADHD: a prospective, randomized, open-label trial. J Am Acad Child Adolesc Psychiatry. 2002;41(7):776-784

30. Pelham WE, Gnagy EM, Burrows-Maclean L, et al. Once-a-day Concerta methylphenidate versus three-times-daily methylphenidate in laboratory and natural settings. Pediatrics. 2001;107(6):e105.
31. Svanborg P, Thernlund G, Gustafsson PA, et al. Efficacy and safety if atomoxetine as add-on to psychoeducation in the treatment of attention deficit/hyperactivity disorder: a randomized, double-blind, placebocontrolled study in stimulant-naïve Swedish children and adolescents. Eur Child Adolesc Psychiatry. 2009;18(4):240-249.

32. Block SL, Kelsey D, Coury D, et al. Once-daily atomoxetine for treating pediatric attention-deficit/hyperactivity disorder: comparison of morning and evening dosing. Clin Pediatr (Phila). 2009;48(7):723-733.

33. Tamayo JM, Pumariega A, Rothe EM, et al. Latino versus Caucasian response to atomoxetine in attention-deficit/hyperactivity disorder. $J$ Child Adolesc Psychopharmacol. 2008;18(1):44-53.

34. Bangs ME, Emslie GJ, et al, (Atomoxetine ADHD and Comorbid MDD Study Group). Efficacy and safety of atomoxetine in adolescents with attention-deficit/hyperactivity disorder and major depression. $J$ Child Adolesc Psychopharmacol. 2007;17(4):407-420.

35. Geller D, Donnelly C, Lopez F, et al. Atomoxetine treatment for pediatric patients with attention-deficit/hyperactivity disorder with comorbid anxiety disorder. J Am Acad Child Adolesc Psychiatry. 2007;46(9):1119-1127.

36. Gau SF, Huang YS, Soong WT, et al. A randomized, double-blind, placebo-controlled clinical trial on once-daily atomoxetine hydrochloride in Taiwanese children and adolescents with attention-deficit/hyperactivity disorder. J Child Adolesc Psychopharmacol. 2007;17:4:447-461.

37. Kratochvil CJ, Vaughan BS, Mayfield-Jorgensen ML, et al. A pilot study of atomoxetine in young children with attention-deficit/hyperactivity disorder. J Child Adolesc Psychopharmacol. 2007;17(2):175-185.

38. Prasad S, Harpin V, Poole L, et al. A multi-centre, randomized, openlabel study of atomoxetine compared with standard current therapy in UK children and adolescents with attention-deficit/hyperactivity disorder (ADHD). Curr Med Res Opin. 2007;23(2):379-394.

39. Arnold LE, Aman MG, Cook AM, et al. Atomoxetine for hyperactivity in autism spectrum disorders: placebo-controlled crossover pilot trial. J Am Acad Child Adolesc Psychiatry. 2006;45(10):1196-1205.

40. Newcorn JH, Michelson D, Kratochvil CJ, et al. Low-dose atomoxetine for maintenance treatment of attention-deficit/hyperactivity disorder. Pediatrics. 2006;118(6):e1701-e1706.

41. Escobar R, Soutullo C, San Sebastián J, et al. Atomoxetine safety and efficacy in children with attention deficit/hyperactivity disorder (ADHD): initial phase of 10-week treatment in a relapse prevention study with a Spanish sample. Actas Esp Psiquiatr. 2004;33(1):26-32. Article in Spanish.

42. Allen AJ, Kurlan RM, Gilbert DL, et al. Atomoxetine treatment in children and adolescents with ADHD and comorbid tic disorders. Neurology. 2005;65(12):1941-1949.

43. Kelsey DK, Sumner CR, Casat CD, et al. Once-daily atomoxetine treatment for children with attention-deficit/hyperactivity disorder, including an assessment of evening and morning behavior: a doubleblind, placebo-controlled trial. Pediatrics. 2004;114(1):e1-e8.

44. Biederman J, Heiligenstein JH, Faries DE, et al. Efficacy of atomoxetine versus placebo in school-aged girls with attention-deficit/hyperactivity disorder. Pediatrics. 2002;110(6):e75.

45. Michelson D, Allen AJ, Busner J, et al. Once-daily atomoxetine treatment for children and adolescents with attention deficit hyperactivity disorder: a randomized, placebo-controlled study. Am J Psychiatry. 2002;159(11):1896-1901.

46. Spencer T, Heiligenstein JH, Biederman J, et al. Results from 2 proof-of-concept, placebo-controlled studies of atomoxetine in children with attention-deficit/hyperactivity disorder. J Clin Psychiatry. 2002;63(12):1140-1147.

47. Michelson D, Faries D, Wernicke J, et al. Atomoxetine in the treatment of children and adolescents with attention-deficit/hyperactivity disorder: a randomized, placebo-controlled, dose-response study. Pediatrics. 2001;108(5):e83.

48. Kahbazi M, Ghoreishi A, Rahiminejad F, et al. A randomized, doubleblind and placebo-controlled trial of modafinil in children and adolescents with attention deficit and hyperactivity disorder. Psychiatry Res. 2009;168(3):234-237. 
49. Amiri S, Mohammadi MR, Mohamaddi M, et al. Modafinil as treatment for Attention-Deficit/Hyperactivity Disorder in children and adolescents: a double blind, randomized clinical trial. Prog Neuropsychopharmacol Biol Psychiatry. 2008;32(1):145-149.

50. Boellner S, Earl CQ, Arora S. Modafinil in children and adolescents with attention-deficit/hyperactivity disorder: a preliminary 8-week, open-label study. Curr Med Res Opin. 2006;22(12):2457-2465.

51. Wigal SB, Biederman J, Swanson JM, et al. Efficacy and safety of modafinil film-coated tablets in children and adolescents with or without prior stimulant treatment for attention-deficit/hyperactivity disorder: pooled analysis of 3 randomized, double-blind, placebo-controlled studies. Prim Care Companion J Clin Psychiatry. 2006;8(6):352-360.

52. Biederman J, Swanson JM, Wigal SB, et al. A comparison of once-daily and divided doses of modafinil in children with attention-deficit/hyperactivity disorder: a randomized, double-blind, and placebo-controlled study. J Clin Psychiatry. 2006;67(5):727-735.

53. Greenhill LL, Biederman J, Boellner SW, et al. A randomized, doubleblind, placebo-controlled study of modafinil film-coated tablets in children and adolescents with attention-deficit/hyperactivity disorder. J Am Acad Child Adolesc Psychiatry. 2006;45(5):503-511.
54. Biederman J, Swanson JM, Wigal SB, et al. Efficacy and safety of modafinil film-coated tablets in children and adolescents with attention-deficit/hyperactivity disorder: results of a randomized, double-blind, placebo-controlled, flexible-dose study. Pediatrics. 2005;116(6):e777-e784.

55. Hansen EH. Technology assessment in a user perspective - experiences with drug technology. Int J Technol Assess Health Care. 1992;8(1):150-165.

56. Gøtzsche P, Hróbjartsson A, Johansen HK, et al. Constraints on publication rights in industry-initiated clinical trials. JAMA. 2006;295(14):1645-1646.

57. Aagaard L, Hansen EH. Information about ADRs explored by pharmacovigilance approaches: a qualitative review of studies on antibiotics, SSRIs, and NSAIDs. BMC Clin Pharmacol. 2009;9:4. 


\section{Appendix I \\ Search strategy: complete databases were searched until February 20I I

Embase
Adverse event
Methylphenidate
Adverse drug reaction AND methylphenidate
(Adverse event OR adverse drug reaction) AND psychostimulant
Atomoxetine OR modafinil OR methylphenidate OR amphetamine
(Atomoxetine OR modafinil OR methylphenidate OR amphetamine)
AND adverse event
PubMed
Adverse event
Methylphenidate
Adverse event AND methylphenidate
Adverse event OR adverse drug reaction
Psychostimulant
(Adverse event OR adverse effect) AND psychostimulant
Atomoxetine OR modafinil OR methylphenidate OR amphetamine
(Atomoxetine OR modafinil OR methylphenidate OR amphetamine)
AND adverse event
PsyclNFO
Adverse event
Methylphenidate
Adverse event AND methylphenidate
Side effect
Adverse drug reaction
Psychostimulant
(Adverse drug reaction OR side effect) AND psychostimulant
Adverse drug reaction OR side effect OR adverse event
Atomoxetine OR modafinil OR methylphenidate OR amphetamine
(Adverse event OR side effect OR adverse drug reaction) AND
(Atomoxetine OR modafinil OR methylphenidate OR amphetamine)

\section{Appendix 2}

\section{Excluded studies listed by reason for} exclusion, alphabetically by first author Meta-analyses

Bangs ME, Tauscher-Wisniewski S, Polzer J, et al. Meta-analysis of suicide-related behavior events in patients treated with atomoxetine. J Am Acad Child Adolesc Psychiatry. 2008;47(2):209-218.

Greenhill LL, Newcorn JH, Gao H, et al. Effect of two different methods of initiating atomoxetine on the adverse event profile of atomoxetine. J Am Acad Child Adolesc Psychiatry. 2007;46(5):566-572.

Kratochvil CJ, Wilens TE, Greenhill LL, et al. Effects of long-term atomoxetine treatment for young children with attention-deficit/hyperactivity disorder. J Am Acad Child Adolesc Psychiatry. 2006;45(8):919-927.
Kratochvil CJ, Michelson D, Newcorn JH, et al. High-dose atomoxetine treatment of ADHD in youths with limited response to standard doses. J Am Acad Child Adolesc Psychiatry. 2007;46(9):1128-1137.

Kratochvil CJ, Milton DR, Vaughan BS, et al. Acute atomoxetine treatment of younger and older children with ADHD: a meta-analysis of tolerability and efficacy. Child Adolesc Psychiatry Ment Health. 2008;2(1):25.

Polzer J, Bangs ME, Zhang S, et al. Meta-analysis of aggression or hostility events in randomized, controlled clinical trials of atomoxetine for ADHD. Biol Psychiatry. 2007;61(5):713-719.

Schachter HM, Pham B, King J, et al. How efficacious and safe is short-acting methylphenidate for the treatment of attention-deficit disorder in children and adolescents? A meta-analysis. CMAJ. 2001;165(11):1475-1488.

Wilens TE, Newcorn JH, Kratochvil CJ, et al. Long-term atomoxetine treatment in adolescents with attention-deficit/ hyperactivity disorder. J Pediatr. 2006;149(1):112-119.

\section{Review articles}

Brams M, Moon E, Pucci M, et al. Duration of effect of oral long-acting stimulant medications for ADHD throughout the day. Curr Med Res Opin. 2010;26(8):1809-1825.

Findling RL. Evolution of the treatment of attention-deficit/ hyperactivity disorder in children: a review. Clin Ther. 2008;30(5):942-957.

Merkel RL Jr, Kuchibhatla A. Safety of stimulant treatment in attention deficit hyperactivity disorder: Part I. Expert Opinion Drug Saf. 2009;8(6):655-668.

Wernicke JF, Faries D, Girod D, et al. Cardiovascular effects of atomoxetine in children, adolescents, and adults. Drug Saf. 2003;26(10):729-740.

\section{Studies with a mixture of adults and children/} adolescents

Bangs ME, Jin L, Zhang S, et al. Hepatic events associated with atomoxetine treatment for attention-deficit hyperactivity disorder. Drug Saf. 2008;31(4):345-354.

Maia CR, Matte BC, Ludwig HT, et al. Switching from methylphenidate immediate release to MPH-SODAS in attention-deficit/hyperactivity disorder. Eur Child Adolesc Psychiatry. 2008;17(3):133-142.

Paterson R, Douglas C, Hallmayer J, et al. A randomised, double-blind, placebo-controlled trial of dexamphetamine in adults with attention deficit hyperactivity disorder. Aust N Z J Psychiatry. 1999;33(4):494-502. 
Wernicke JF, Holdridge KC, Jin L, et al. Seizure risk in patients with attention-deficit-hyperactivity disorder treated with atomoxetine. Dev Med Child Neurol. 2007;49(7):498-502.

\section{Sub-group analyses of clinical studies}

Durell TM, Pumariega AJ, Rothe EM, et al. Effects of openlabel atomoxetine on African-American and Caucasian pediatric outpatients with attention-deficit/hyperactivity disorder. Ann Clin Psychiatry. 2009;21(1):26-37.

Spencer TJ, Sallee FR, Gilbert DL, et al. Atomoxetine treatment of ADHD in children with comorbid Tourette syndrome. J Atten Disord. 2008;11(4):470-481.

\section{ADRs presented as number of children reporting ADRs, assessment of rate not possible}

Davari-Ashtiani R, Shahrbabaki ME, Razjouyan K, et al. Buspirone versus methylphenidate in the treatment of attention deficit hyperactivity disorder: a double-blind and randomized trial. Child Psychiatry Hum Dev. 2010;41(8):641-648.

Barkley RA, McMurray MB, Edelbrock CS, et al. Side effects of methylphenidate in children with attention deficit hyperactivity disorder: a systemic, placebo-controlled evaluation. Pediatrics. 1990;86(2):184-192.

Firestone P, Musten LM, Pisterman S, et al. Short-term side effects of stimulant medication are increased in preschool children with attention-deficit/hyperactivity disorder: a double-blind placebo-controlled study. J Child Adolesc Psychopharmacol. 1998;8(1):13-25.
Pelham WE, Gnagy EM, Chronis AM, et al. A comparison of morning-only and morning/late afternoon Adderall to morning-only, twice-daily, and three times-daily methylphenidate in children with attention-deficit/hyperactivity disorder. Pediatrics. 1999;104(6):1300-1311.

Pelham WE Jr, Greenslade KE, Vodde-Hamilton M, et al. Relative efficacy of long-acting stimulants on children with attention deficit-hyperactivity disorder: a comparison of standard methylphenidate, sustained-release methylphendiate, sustained-release dextroamphetamine and pemoline. Pediatrics. 1990;86(2):226-237.

Pelham WE, Aronoff HR, Midlam JK, et al. A comparison of Ritalin and Adderall: efficacy and time-course in children with attention-deficit/hyperactivity disorder. Pediatrics. 1999;103(4):e43.

Rugino TA, Copley TC. Effects of modafinil in children with attention-deficit/hyperactivity disorder: an openlabel study. J Am Acad Child Adolesc Psychiatry. 2001;40(2):230-235.

Swanson JM, Greenhill LL, Lopez FA, et al. Modafinil filmcoated tablets in children and adolescents with attentiondeficit/hyperactivity disorder: results of a randomized, double-blind, placebo-controlled, fixed-dose study followed by abrupt discontinuation. J Clin Psychiatry. 2006;67(1):137-147.
Neuropsychiatric Disease and Treatment

\section{Publish your work in this journal}

Neuropsychiatric Disease and Treatment is an international, peerreviewed journal of clinical therapeutics and pharmacology focusing on concise rapid reporting of clinical or pre-clinical studies on a range of neuropsychiatric and neurological disorders. This journal is indexed on PubMed Central, the 'PsycINFO' database and CAS.

\section{Dovepress}

The manuscript management system is completely online and includes a very quick and fair peer-review system, which is all easy to use. Visit http://www.dovepress.com/testimonials.php to read real quotes from published authors.

Submit your manuscript here: http://www.dovepress.com/neuropsychiatric-disease-and-treatment-journal 